This article was downloaded by: [Malmö högskola]

On: 29 April 2013, At: 06:46

Publisher: Routledge

Informa Ltd Registered in England and Wales Registered Number: 1072954 Registered office: Mortimer House, 37-41 Mortimer Street, London W1T 3JH, UK

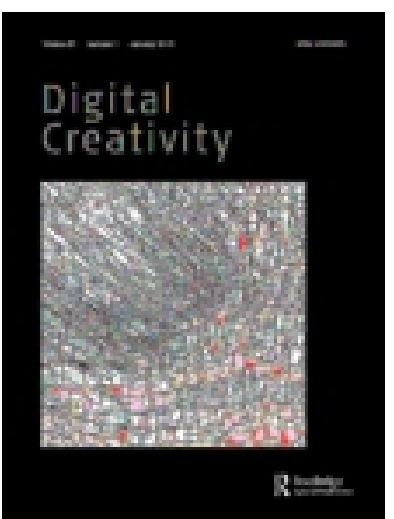

\title{
Digital Creativity
}

Publication details, including instructions for authors and subscription information: http://www.tandfonline.com/loi/ndcr20

\section{Embodied imagination: a hybrid method of designing for intimacy}

Lone Koefoed Hansen ${ }^{a}$ \& Susan Kozel ${ }^{b}$

${ }^{a}$ University of Aarhus, Denmark

${ }^{\mathrm{b}}$ Simon Fraser University, Canada

Published online: 02 Nov 2007.

To cite this article: Lone Koefoed Hansen \& Susan Kozel (2007): Embodied imagination: a hybrid method of designing for intimacy, Digital Creativity, 18:4, 207-220

To link to this article: http://dx.doi.org/10.1080/14626260701743200

\section{PLEASE SCROLL DOWN FOR ARTICLE}

Full terms and conditions of use: http://www.tandfonline.com/page/terms-and-conditions

This article may be used for research, teaching, and private study purposes. Any substantial or systematic reproduction, redistribution, reselling, loan, sub-licensing, systematic supply, or distribution in any form to anyone is expressly forbidden.

The publisher does not give any warranty express or implied or make any representation that the contents will be complete or accurate or up to date. The accuracy of any instructions, formulae, and drug doses should be independently verified with primary sources. The publisher shall not be liable for any loss, actions, claims, proceedings, demand, or costs or damages whatsoever or howsoever caused arising directly or indirectly in connection with or arising out of the use of this material. 


\title{
Embodied imagination: a hybrid method of designing for intimacy
}

\author{
Lone Koefoed Hansen ${ }^{1}$ and Susan Kozel ${ }^{2}$ \\ 1 University of Aarhus, Denmark \\ 2 Simon Fraser University, Canada \\ koefoed@multimedia.au.dk; susan_kozel@sfu.ca
}

\begin{abstract}
Situated in the domain of research into mobile, wireless, networked and wearable computing, this exploratory paper introduces the embodied imagination method and explains how it can contribute to the design process by creating an elastic space of performance that incorporates daily life and personal imagination into the design process. It is based on a study called Placebo Sleeves which was an experiential design phase of a larger project in wearable computing called whisper[s]. The innovation offered by this research is twofold: an integration of previously distinct methodologies, and an interdisciplinary theoretical framework relevant to the design of devices for affective, networked communication. The methodologies are shaped both by user experience models and by performance practices. We also articulate a domain of public dreaming, located at the conjunction of the private, public and secret within human existence, and suggest that shared use of mobile technologies has the potential to be situated there.
\end{abstract}

Keywords: affective computing, mobile computing, participatory design, performance, phenomenology, social computing, wearable computing

\section{Research paradoxes}

The movement toward incorporating technologies intimately into everyday experiences is gaining momentum. Sports, entertainment, medicine, fashion, personal communications and personal data organization are being transformed by technological advancements in wireless protocols, conductive materials, miniaturisation of circuitry and flexible long-life batteries ${ }^{1}$. Frequently, this research is based on the conviction that people's lives can be augmented and improved by drawing technologies closer to the body, or it is simply fuelled by the desire to sell us more gadgets. This paper is written from the premise that it is important to investigate the social, performative and phenomenological aspects of technological embodiment. This amounts to widening the range of methods and concepts relevant to the design of mobile, intimate and personal technologies. A further premise is that the ever expanding technological culture in the consumer economies of the East and the West needs the analyses and critiques offered by collaborations between design professionals and practitioners of creative embodied arts in order to avoid narrow or anachronistic approaches to the next generation of technological artifacts, particularly in the domain of affective or intimate computing. This paper discusses current methodologies for envisioning and designing emerging technologies, then introduces a study called Placebo Sleeves. With this study we attempted the difficult task of investigating technologies not yet known and applications as yet undefined by the worlds of art, design 
and the hardware and software industries. The study was conducted through a method we call embodied imagination, a creative integration of experiential practices from the worlds of participatory design and performance. What is offered below is an account drawn from the early stages of the experiential parts of a longer and more complex project.

Researchers are often driven by the possibilities proposed by new technologies in a feedback loop between technological functionality and human needs. It is common for investigations, workshops and design processes to correspond to a design proposal. However, when investigating the implications of new people-technology relations we face important and fundamental questions: How do we determine and understand as yet unexpected and unanticipated needs? How do we address the physical and social consequences that arise as digital technologies are drawn into daily life, often literally disappearing into clothing and onto bodies? The design challenge is that we are limited not just by the functionality of current technologies but by our shared cultural attitudes and expectations permeating these technologies. There are assumptions implicit to our design processes that may constrain us but at the same time be invisible to us.

In order to work around the paradox of implementing a design process that questions its own assumptions, it can be beneficial to change the modality of research from focusing on 'implications for design' to exploring connections and prospective uses we cannot yet envision. In this paper we will argue that theories and practices from performance and phenomenology can enable researchers to stage an open ended process where participants - without focusing on outcomes - can explore their embodied imagination as it unfolds through their lived experience of daily life and release the innovation latent in these embedded, embodied contexts.

\section{$2 \quad$ Living with and through technology}

It is not unprecedented for new technologies to create new needs, and vice versa, but since we are now witnessing fundamental changes in the materiality and ubiquity of computers current research in particular can benefit from investigating the phenomenological dimensions of these changes. The relation between people and technology becomes highly relevant when computing is incorporated intimately into daily experiences, to the extent of being carried with us everywhere, woven into clothing, or worn directly on the skin. More than ever, we live with and through our technologies. Acknowledging the sensory, affective, poetic and corporeal qualities of the moment of lived experience is key to designing and understanding the next generation of technologies - and this is not always afforded by existing design methodologies. The social and intimate dimensions of life are even more important once new technologies cause communication modalities currently dominated by verbal and visual paradigms to include body language and physical or latent layers of communication. The emphasis offered in this paper is not just on the body for the sake of it, or because it seems to be trendy. We believe that expanding and enriching the design of future technologies is reliant upon a deeper understanding of the social connectedness of our lives, and this connectedness occurs through the senses.

Theatre scholar Alan Read indicates that an ethics of theatre affirms

the power of theatre to affect life, emotionally and biologically, and with this belief in theatre there is responsibility to ask how it does this and to what purpose.

(Read 1995, p.89)

It is clear that the word 'theatre' in this phrase can easily be replaced by the word 'design'. Once the creation of work is regarded from an embodied or an ethical perspective it is 


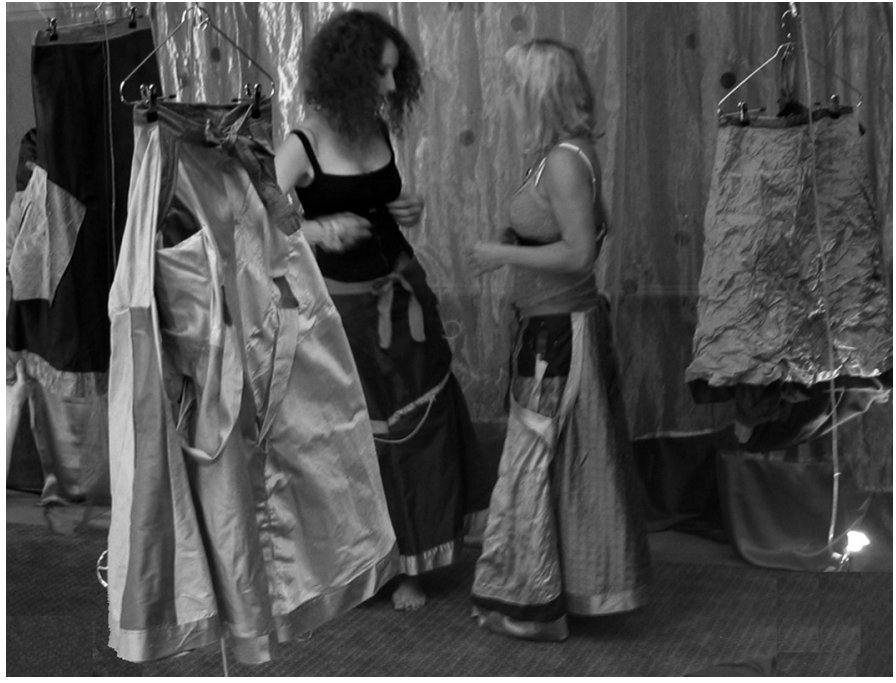

Figure 1. Exhale, an iteration of the whisper[s] project, at Emerging Technologies, SIGGRAPH 2005.

impossible to overlook its social and political implications. Technology is not just about computers, and performance techniques do not just occur on stage. Both are about communication across people in a rapidly advancing technological age where the relations between bodies, design, art, technologies and the marketplace need to be continuously evaluated and, when necessary, changed.

\subsection{Context: wearables and systems for embodied communication}

Wearable computing and the expansion of mobile wireless communications are examples of not just living with, but living through, technologies. This dual approach, which recognises that our communication is not just facilitated by our devices but is transformed by them, is evident in various projects investigating new ways of sending and receiving text messages (SMS). Amongst them is Hug Shirt by CuteCircuit, a t-shirt that "allows people to exchange the physical sensation of a hug over distance" by operating as a Bluetooth accessory for Java-enabled mobile phones. The sex industry is, of course, also investigat- ing the convergence of wireless embedded technologies and bodies, one example is The Toy, a very small and Bluetooth-enabled vibrator, aimed at creating an "intimate, silent connection between two lovers, regardless of distance". While both Hug Shirt and The Toy enable embodied communication, neither of the projects employs fundamentally new ways of communicating: both rely on a specific 'send' command, in this sense they coincide with the SMS model.

The Placebo Sleeves study presented in this paper emanates from whisper [s] - an ongoing wearable/wireless research platform exploring alternative communication possibilities which attempts to get beyond the SMS model of communication (Kozel 2006; Schiphorst 2006). whisper [s] explores possible future communication protocols where connectivity between people is more than flashy gadgets with large bandwidth (Figure 1). The project is about the future of social and affective computing where technology can contribute, not just to the functional, but to the expressive and emotional texture of people's lives. Using biometric sensors, haptic and audio actuators, micro-controllers and wireless networking applications embedded in custom designed clothing, whisper [s] affords participants the ability to create interpersonal connections by mapping the physiological data (e.g. breath and heart rate) of one person onto linked and networked devices embedded in clothing of other people: a respiration sensor in a top can activate wirelessly a vibrator in a skirt, causing one person's breath to make the lining of another person's skirt vibrate. This other person can respond by communicating body data synchronously to the first person, or by mapping it outward to someone else or to a group. This is breathing between bodies.

The primary presentation modality of the whisper [s] project has been the public installation, and the garments have been large: elaborate skirts or jackets containing reasonable amounts of hardware and batteries (Figure 2). 


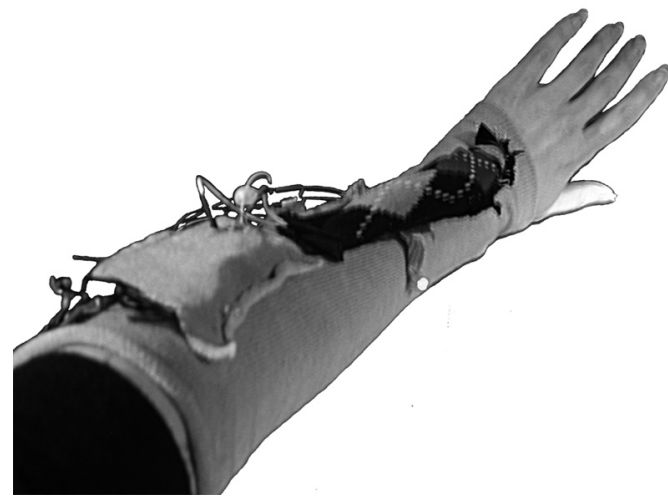

Figure 2. Students affiliated with the whisper[s] project integrated the electronics of whisper[s] into a sleeve. As evident in the above image, it turned out pretty bulky.

In contrast, Placebo Sleeves was based deliberately around less defined objects that could evoke desire and imagination. We unspooled the design process by constructing 'sleeves' without technology, but with a distinct poetic look and feel. This allowed us to explore the scope for coupling technology with bodies in smaller clothing items and to investigate the possibility for creating a more elastic space of performance with the potential for stretching beyond the public art installation and into people's daily lives.

\section{Researching embodied technologies}

Prior to the elaboration of the Placebo Sleeves study it is useful to consider the goals and traditions that informed our integrated research method. The embodied imagination method is less concerned with getting input for a concrete design proposal than it is with integrating the body directly into the loop of design iteration in an open ended way. The starting point for the embodied imagination approach and the Placebo Sleeves study was the development cycle of the whisper [s] project within which small design iterations, including user-experience workshops in thea- tre settings, were integral to the research process. These workshops gathered participants in a theatre space complete with lighting, music and some basic stage design. They were guided in a series of movement improvisations wearing the whisper [s] garments embedded with varying degrees of active technologies and their reactions were documented in various ways (Schiphorst and Andersen 2004). With Placebo Sleeves we wanted to continue these qualitative, embodied and performative research methods, but also to shift and transform them slightly. Two things were key:

1 extending outwards from the contained imaginative context of the theatre to enhance the performativity of everyday life, and

2 not assuming in advance what physical or affective information participants wanted to communicate (i.e. not confining the interaction to the exchange of breath and heart).

The embodied imagination method was the result of these considerations and was enacted by 'installing' poetic objects in people's lives in order to access their dreams, desires and reflections on technologies intimately incorporated into everyday experiences. Some design methods try to obtain as many ideas as possible, others try to understand the user's context, and still others are interested in testing prototypes. We were interested in people's relations to embodied technologies as analysed or simply interpreted through their actions and reflections. The data was valuable to us for being more evocative and poetic than functional. A slice of methodological history is useful prior to the detailed description of the study.

\subsection{Foundations in design}

As technology pervades the domestic domain, designing for use settings is increasingly a matter of designing for people's lives. This has triggered a refinement of methods investigating users' knowledge and imaginations based on their own lives. When inviting people to integrate their imaginations into a design proc- 
ess there are, broadly stated, two approaches: you can bring the project space to the people or the people to the project space (Muller 2003).

When researchers invite participants to a lab, the designers are in control of the surroundings and often bring props like cardboard, foam, pictures and other materials suitable for shaping provisionary mock-ups. Meeting rooms can be designed specifically for innovation and generation of ideas but 'the lab' is also where designers bring their props and processes to the participants' surroundings for a workshop. In a broader sense, the lab works like a performance space and can be seen in interface design and evaluation methods like focus groups, inspiration card workshops (Halskov and Dalsgård 2006), scenario making and future workshops (Greenbaum and Kyng 1991; Brandt 2006).

When project space is brought to the participants, the designers insert objects into the participants' lives. Since Gaver and others introduced the situationist inspired self-report packages of Cultural Probes, the method has been widely acknowledged as being a fruitful (and fun) way of investigating people's lives in order to come up with new designs. A package containing a camera, carefully chosen artifacts and tasks enables the participant to perform a life for the designer. The related Technology Probes (Hutchinson et al. 2003) narrow the investigation scope by installing a somewhat functional artifact in participants' homes. The purpose is to investigate participants' relations to the artifact's function and presence with respect to social, engineering and design issues.

In our case, we decided to use sleeves with a specific, poetic appearance in order to invoke people's embodied imaginations, and this decision rules out both cultural and technological probes. Unlike the Cultural Probes approach we have a designed object, but unlike the Technology Probes approach we know neither the use nor the specific technology.
Dunne and Raby's Placebo Project (Dunne and Raby 2001) is a third option, and is obviously relevant because of the designation 'placebo'. Dunne and Raby created eight objects and told participants that the objects had (or could have) a specific function. Participants adopted an object into their homes for a month with the purpose of invoking curiosity and imagination. The purpose was not to test the objects but to get participants to reflect on the objects' signifying properties. In this way Dunne and Raby staged participants' reflection through their curiosity towards the design. Further, participants' homes were transformed into a performance stage. Dunne and Raby's Placebo Project comes close to addressing our needs: the design frame is distinct (participants are informed of a functionality) but is open for reflective imagination (participants are not given details). Also, the participant's relation to the artifact is carefully staged both through the set-up of the experiment (objects are adopted) and through the specific design. However, we are interested in making people performers in their lives instead of transforming their home into a performance stage and as such our methodology needed to integrate a phenomenological or self-reflective dimension. So if we combine the Cultural Probes method with Dunne and Raby's Placebo Project method, we then have the carefully staged non-functional object placed in the participant's life, making the participant a performer in her own life. This is the design heritage of the embodied imagination method.

\subsection{Foundations in performance and phenomenology}

The embodied imagination method integrated design techniques with key elements drawn from performance and phenomenology. A premise of embodied imagination is that the merging of technologies and bodies need not be on a spectacular or dramatic level, it can occur on a pedestrian, daily level which emphasises not just the practice of everyday life, 
but the performativity of everyday life (Amin and Thrift 2002; Certeau 2002). Performance encompasses intimate, playful and even banal or ambiguous gestures as conduits for thoughts and emotions.

Our definition of performance is a triangulation across theories from gender studies, locative media and performance studies. Philosopher Judith Butler constructed a (now classic) argument that maleness and femaleness in society are not based on biology but on actions, supporting the claim that gender is performative and is enacted on a moment by moment basis throughout our lives (Butler 1989). Approaches to media architecture such as locative media, suggest that portable, wireless, mobile devices afford people the possibility to become "performers in their own environments" (Haque 2002). And of course, performance studies respects the breadth of performativity: from sports, to art, to professional identities, to ritual (Schechner 2003). While the theoretical framework for Placebo Sleeves is based on an appreciation of arguments that all acts are performative, the method required a slightly more specific framing of performativity. Basic techniques from theatre and performance were implemented with the hope that these would spill over into the lives of our participants. Our framing was highly deliberate and qualitative, it consisted in

1 imbuing the sleeves with specific textural and aesthetic qualities,

2 creating a special performative ambience around the distribution of the sleeves, and

3 generating a fluid, or we like to say 'elastic' space of performativity around the wearing of the sleeves and the documenting of actions, thoughts and experiences. Existential phenomenology, defined as a descriptive methodology that avoids preconceptions in order to return to the data derived from the moment of lived experience, is central to the embodied imagination approach. Methods for phenomenological reflection work together with performance to make up the uniqueness of this approach. A phenomenological approach relies on refining techniques for reflecting on an experience while in the midst of that experience. The premise is that human beings can only reflect on the world from within the thickness of the world, not from an external vantage point, and as such perception is always embodied (MerleauPonty 1987; Varela et al. 1993; Kozel 2007). It is somehow appropriate that embedded technologies be considered from the vantage point of embedded perception and reflection. These reflections can take the form of language, images, sounds or drawings, and they point to the multi-sensory and conceptual richness of the experience. Further, phenomenology is based on the untenability of a distinction between mind and body (Merleau-Ponty 1987; Damasio 1994) ${ }^{2}$.

The space for phenomenological reflection was implicitly tied to theatre techniques and mechanisms for improvisation and, above all, for escaping habit. Theatre constructs a context for both audience and actors to leave their habitual environment and visit an unknown territory. From the practice of theatre we borrowed the skills for creating a space of permission, an environment where attention, awareness and imagination could be co-mingled. The Placebo Sleeves study had participants enter a space where habits of expression and conventional use of devices could be suspended, so that different behaviour and expression could occur. We hoped that once they left this physical space and re-entered their lives some of the sense of 'bracketing out' biases and expectations around technologies might be maintained so that new experiences could emerge and be reflected in their personal documentation. The act of wearing the sleeves created a context allowing for participants' improvisations within their daily lives: where improvisation consists in acting spontaneously without concern for pre-established forms and without these actions being judged as right or wrong (Boal 1992). It is worth noting that 
we did not employ a storytelling technique, we quite deliberately set a more open ended context for affective, gestural and expressive improvisation.

\section{The Placebo Sleeves study \\ 4.1 Basic materials: sleeves and note- books}

Sleeves were constructed out of a range of materials (Figure 3). Emphasis was placed on texture and colour. All sleeves were handmade with decorative stitches; some were knitted and some were sewn, in widths ranging from wrist bands to full arm's length. The colours, textures and patterns were intended to be playful. Knowing that we would have 12 participants we constructed 20 sleeves, thus affording participants the ability to choose, immediately investing a bit of their personalities into the process. We created some in sombre colours for those who might prefer more subtle accessories, some in elaborate 70 's prints, and some made out of modified women's tights for an evening gown or fancy dress, feel. Most sleeves were reversible. All sleeves were characterised by two elements: each had a whisper[s] logo stitched into it, a simple sketch of a human form with the heart articulated in contrasting thread; and each had a tactile prosthetic lump embedded in them somewhere near the wrist.

This lump was our approximation of 'something living in the sleeve', this metaphor we transposed from one of the earlier

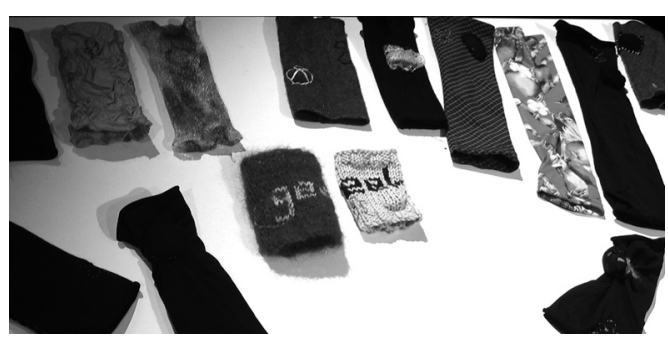

Figure 3 . The sleeves lying on the table waiting to be selected.

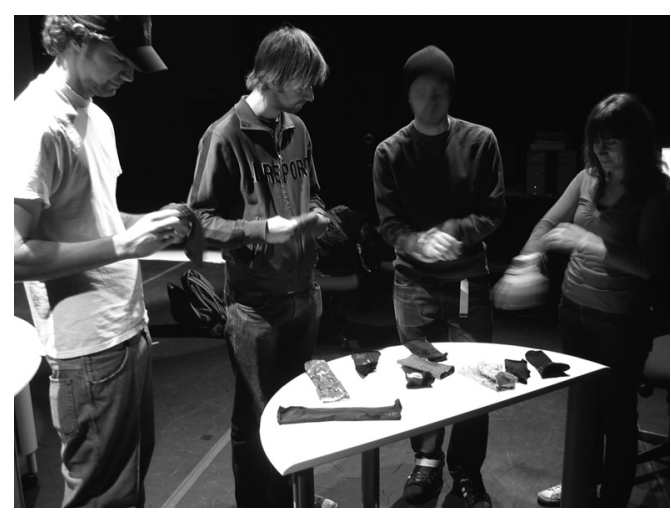

Figure 4. Participants selecting sleeves in the theatre setting.

whisper [s] workshops. It was also an obvious placebo for technology, as if it were a sensor, a battery or a small bit of circuitry. The lump was a particularly gooey and touch-inviting piece of silicon; formerly a stress ball, it was carved up into segments and stitched into the sleeves. In its most benign connotation it was suggestive of an on/off switch, more creepily it was like a lump of flesh, one's own or another's.

Participants were asked to collect the sleeves at one of two locations. The primary location was a black box performance space attached to a lab. Theatrical lighting was used, darkening the space while illuminating the tables holding the selection of sleeves (Figure 4). They were invited into the staged space and asked to select a sleeve. This took some time, for much trying on, touching, feeling and moving of arms occurred. It seemed as if the sleeves were 'tested' in some way, for comfort, for fit, but, above all, for a sense that they might be a pleasing addition to the participants' bodies for the next four days. This distribution was an event, carefully staged in order not exactly to give the artifacts a particular meaning, but to imbue them with potential, and to create conditions for imagination, dreams or fantasy. The subtle uses of theatricality at this stage of the process fostered the participants' awareness of the artifacts' lack of 


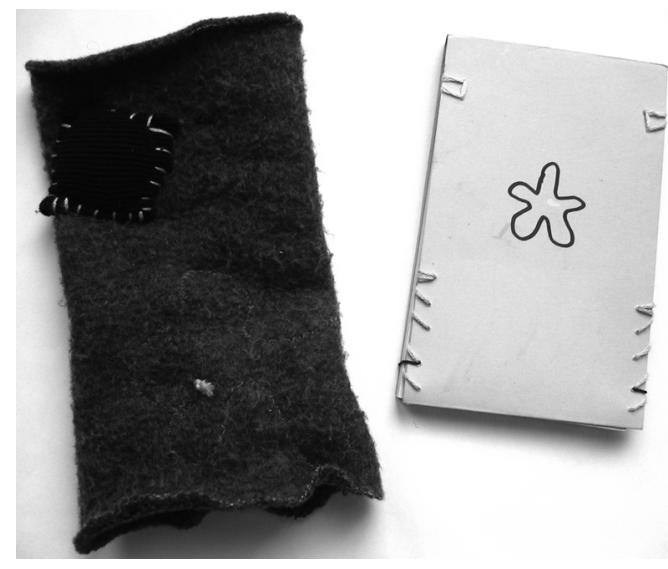

Figure 5. A sleeve and a notebook selected by a participant.

function and that they were, to some degree, performers in their own lives, performing and inventing connections with others which were both imaginary and embodied.

Participants also chose one of 14 basic paper notebooks modified with stitching and drawing so that each was consistent with the aesthetic of the study (Figure 5). Instructions inserted into the book charted a balance between providing information and creating a poetic framework for an open-ended set of experiences. We did not want to prefigure the results or over-determine the procedures, but still found certain pieces of information vital such as what was communicated, to whom and when. In the spirit of improvisational theatre techniques, we provided a loose script (Figure 6) but not a story: this was open system scripting not storytelling. Other methods do the opposite, they engage people in design by making a story-driven environment (Dindler et al. 2005). Our script was devised with the intent to create a space for participants to speak from their bodies and imaginations, poetically or functionally, and to create a tone for phenomenological reflection.

No contact was maintained with participants while they lived with the sleeves for four days. This was a waiting phase, after

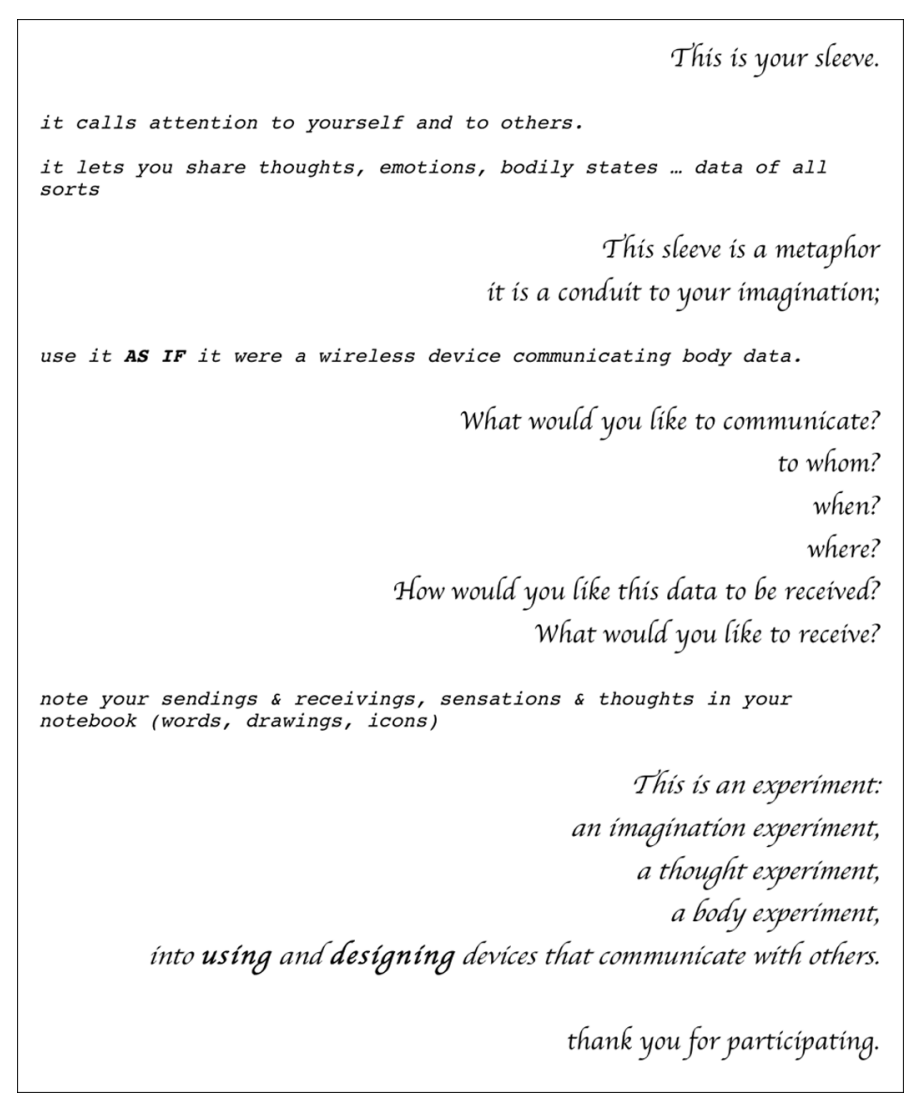

Figure 6. The instructions inserted in the notebooks given to participants together with the sleeve.

which the booklets and sleeves were collected. For reasons of practicality we did not stage a retrieval event, an unfortunate sacrifice because the open performance of the sleeves might have yielded a meaningful denouement. Instead, we made efforts to accommodate people's busy schedules and arranged several collection points.

\subsection{Interpretation and analysis}

The material recorded in the books was remarkably personal, honest, direct, humourous and insightful. These were not fictional narratives or fantasies using personas or characters; they were direct attempts to express hopes, fears, emotions, desires and concerns, combined with some direct suggestions for 
interface development or sensor choice: in other words, all the 'stuff' of human connections. These were glimpses of everyday life. They were not 'the truth' but they were subjective partial truths, the very richness of phenomenology is that it has resonance, rather than positing truth claims. These experiential mini-phenomenologies were written in different styles: descriptive prose, point form, narrative, plus drawings and sketches.

Very few specific technological recommendations or wishes were expressed in the notebooks, the focus was instead on describing social, physical and communicative configurations, and sometimes on how networked presence could be expressed through the sleeve. Participants did not want the technologies to be invisible, but were happy to be aware of wearing and interacting with the sleeves. Surprising to us was that, on the whole, participants would be happy with fewer rather than more interaction possibilities in their sleeves. This could reflect Sterling's ironic account of how gizmos makes end-users "balance on the edge of complexity and utter chaos" (Sterling 2005). The Placebo Sleeves participants clearly did not desire complex functionality.

\subsubsection{Social networks kinaesthetically medi- ated}

There was a strong sense of people living within social and familial networks, and of the urban or geographical space they inhabited. One participant missed her mother and wanted to convey a sense of mountains (Figure 7). One wanted his sleeve with him when he ventured into the centre of the city and felt vulnerable when he left it behind. The sleeves acted as conduits between people but also transmitted environmental information through the bodies that wore them: it was a physical sense of mountains not just mountains, it was a physical need for comfort when venturing into a large city from the suburbs and not just urban space in itself. The tactile, kinaesthetic and affective were foregrounded.

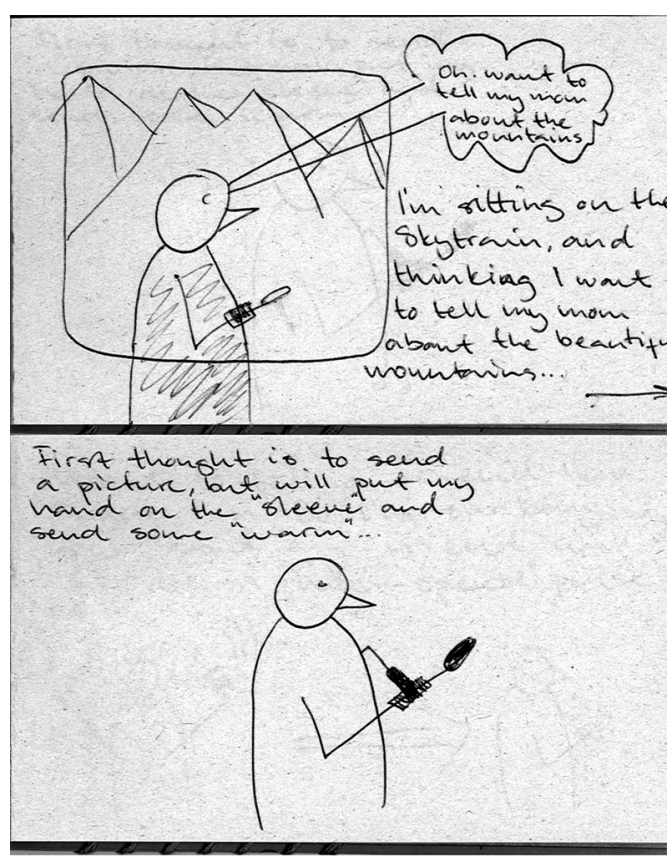

Figure 7. Two notebook pages in sequence revealing the interface between the private and the public.

\subsubsection{Public dreaming}

The interpretation and analysis phase of the study revealed the need for a third term to complement and deepen the embodied imagination method and the activation of an elastic space of performance: public dreaming. A term from performance studies coined by Richard Schechner (Schechner 2003), public dreaming is the state that occurs at the cross-sections of the domains of "the public, the private and the secret" (Schechner 2003, p. 265) (Figure 8). It is a place of negotiation between the three. The secret is of particular significance, for it exists as a shadow of the private and the public. Its existence is pointed to, alluded to, or circumscribed, rather than defined or presented. Schechner demonstrates that theatre, performance and ritual across cultures and over the centuries have addressed the secret on many levels: physical, social, spiritual and therapeutic. The Placebo Sleeves 


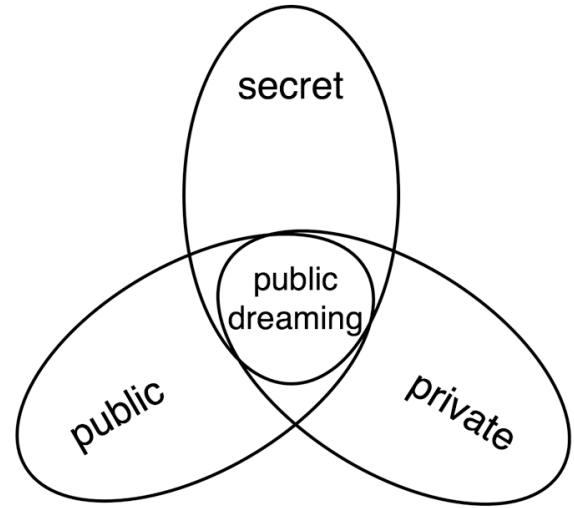

Figure 8. Model depicting different domains: public, private and secret. Public dreaming is a place of negotiation between the three domains. (Model developed from (Schechner 2003)).

project only just grazed the surface of these much larger questions, but did so in a way that could not be overlooked.

Public dreaming, as it was enacted by the participants, was at the same time a collective and individual state. It enabled us, the researchers, to witness the existence of the secret even though we were excluded from its content. As researchers, we too participated in the public dreaming of the project: we were located in the public domain, but the books gave us privileged access to some private moments, and pointed to the secret. We staged and set the tone for the engagement, it was obvious that we constructed the sleeves and booklets by hand, and that we would read the books. In some respects, the books seemed to be written for us. We were regarded as performers in the elastic space of the project, we were not just neutral observers.

\subsubsection{The power of the non-technological} Nobody objected to the absence of technology in their sleeves. We created an analogue experiment and it was taken as such. By providing participants with something that was not technological at all, we invited them to invest their imagination instead of directing their critical capacities towards the functionality of the object. The role for technology was hypothetical, it was presented as a future possibility rather than a present constraint. Technology for this project lived in the space of public dreaming with its potential to mediate the secret, the private and the public.

A significant percentage of the participants expressed regret at having to return the sleeves at the end of the experiment. Further, we noted distinct anthropomorphic tendencies: people gave the sleeves names and attributed personalities to them. They were present as things to engage with. One participant expressed that the "placebo would be addictive" if he could feel his friends' emotions, and vice versa, through it (Figure 9). Returning once more to the state of public dreaming, the example of addiction has roots in public, private and secret. The psycho-physical state of addiction has a public resonance to it (i.e. addiction to popular culture) but also occurs on the levels of the private and the secret. The private implies that we might share details of our addiction with a few close friends, and it contains tones of trust and vulnerability. The secret alludes to a deeper level within the bodies and psyches of each of us, a 'secret' domain that is generally not revealed, possibly not even fully to the person him/herself. The secret, with its implied depths of pain but also pleasure, acts as the lining of our more external experiences. Mobile technologies now comfortably

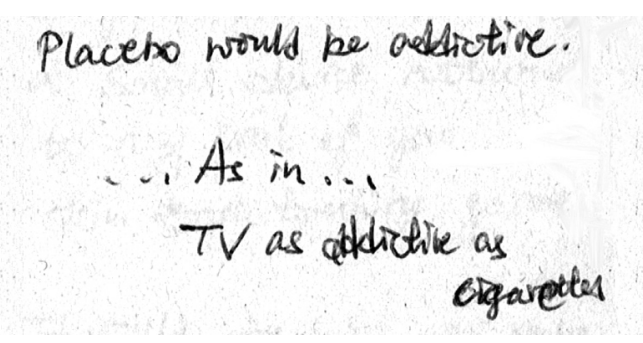

Figure 9. Segment of notebook page hinting at the possibility of an interface between the secret and the public. 

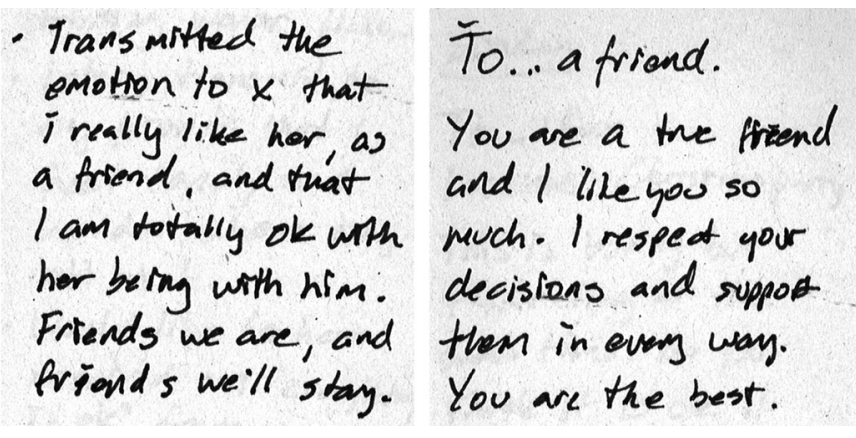

Figure 10. Two notebook pages revealing the domain of the private.

occupy a place that transitions easily between private and public, to the extent of eroding this distinction. The distinction in play now is between private/public and secret, and the question is whether we want the secret to be open to dissemination and exposure, or whether this is even possible.

\subsubsection{Designing the secret}

The Placebo Sleeves study revealed the domain of the secret but did not mine its data or import it into either the public or the private. The study was consistent with the broader goals of the whisper [s] project, which can be articulated as design for intimate computing simultaneous with respect for the personal and affective. We received a considerable level of intimate information (by which we mean private rather than sexual). In one instance complex layers of private feelings about personal relationships were revealed through the presence of the sleeve (Figure 10). One participant "transmitted the emotion", almost like a spontaneous mental hug. Perhaps the emotions were not even on the surface of the participant's mind until he realized he had a 'device' to communicate them.

The importance of the secret continued to catch us by surprise. Two participants made direct references to taking the sleeve off - there were moments, generally intimate moments, when they did not want to wear it (Figure 11). This could not be simply mapped onto sexual behaviour because another participant found the sleeve a nice addition to sex. According to our interpretations, this is where the domain of the private gave way to the domain of the secret (or "too private": Figure 11). The act of removing the sleeve because it felt like it intruded on an intimate situation introduces the important question of whether wearable technologies can be counter-intimate in certain contexts, and whether it is necessary for designers to be aware of a boundary between secret life and performing one's life. By taking off the sleeves, did the participants also suspend the performative mode that we had placed them in, or did the performances simply become secret too? As indicated above, the role of the secret in public dreaming became crucial to the study, acting effectively as the sleeves' off-switch.

The evidence of a 'secret' domain within the state of public dreaming was particularly compelling but may, by its nature, always elide definition. At the same time, the secret plays a key role in the development of future generations of intimate computing as it exists as a shadow of the private and the public. Its existence is pointed to, alluded to, or circumscribed rather than defined or presented. Like the ghost in the machine, the secret respects

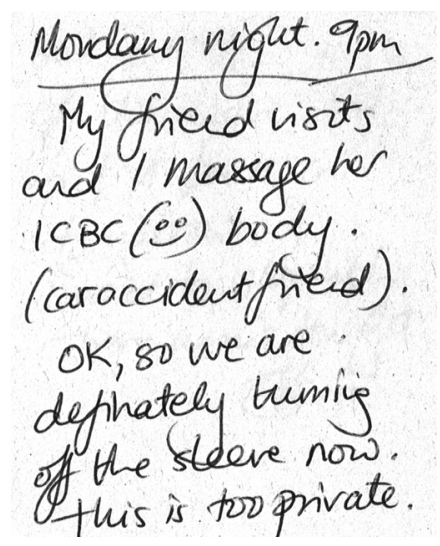

Figure 11. Notebook page revealing a need for a domain of the secret by pointing at an interface between the private and the secret. 
the roles for the ambiguous, illogical or simply baffling in computation and is as such describing the intriguing balance between the rational and emotive dimensions of being human.

\section{Conclusion}

This paper has shown how, through the use of the critical design practice of embodied imagination, the Placebo Sleeves study staged and investigated people's desires, imaginations and reflections on hypothetical embodied technologies. Participants were invited to explore the way they currently and potentially live with and through technologies.

When exploring new paradigms in future technologies, the challenge is to not let current paradigms shape the design of new objects: how can it be new if shaped in the form of the old? If killer apps creating new cultural paradigms are not invented 'centrally' but emerge from culture itself (Rheingold 2002), research into emerging technologies can benefit from investigating emerging cultures according to an open-ended process. Where ethnographic methodologies excel at studying current cultures, performance and theatre methodologies have a long tradition of studying imaginative cultures making them ideal for studying people's imaginations and not their specific actions.

In the Placebo Sleeves study, the creation of an elastic performance space containing participants, their lived lives, the analogue objects and us (the researchers) allowed for the embodied imagination to flow. Participants' imaginative resources were invoked precisely because we worked with poetic and not technological possibilities, with poetic placebos instead of paradigm-reinforcing prototypes. We analysed micro-identities, the "microbelike, singular and plural practices" by layering the spatial with affective, kinaesthetic, gestural and expressive practices (Varela 1999; Amin and Thrift 2002; DeCerteau 2002) instead of being distracted by the functionality of bulky technology, prototypes or electronics.

We created a form of public dreaming, where participants inhabited an elastic space of performance that in some ways made them performers in their own life. We caught a glimpse of people's embodied relationships to technology, communication and interpersonal connections. These lives do not only contain logical and cognitive qualities but are highly affective, physical, passionate and imaginative. As an enactment of our methodological considerations, the Placebo Sleeves study discovered the existence of the secret-existing as a shadow of the private and the public. The secret respects the roles for the ambiguous, illogical or simply baffling in computation and considering how the participants used and reflected on the poetic objects, the secret seems to be important when designing for technologies intimately incorporated in everyday experiences.

\section{Notes}

${ }^{1}$ For current developments in mobile and often wearable technologies see the music and training manager Nike + iPod Sport Kit, personal medical sensing devices by Thought Technologies, Philips' far-future project Skin Probes, CuteCircuit's Hug Shirt and HP's vision of a wireless hub in a watch.

${ }^{2}$ Explicit instructions for doing a phenomenology are not offered in this paper but can be found in (Kozel 2007). The intention with Placebo Sleeves was to keep the expectations and procedure underdefined in order to invite participants to develop their own voices in the notebooks.

\section{Acknowledgements}

We would like to thank the participants for providing us with interesting data, the two anonymous reviewers for providing very useful comments and suggestions, the whisper [s] project (http://whisper.iat.sfu.ca) at the Interactivity Lab of Simon Fraser University in Brit- 
ish Columbia, Canada and also the research project The Aesthetics of Interface Culture at University of Aarhus supported by The Danish Research Council for the Humanities.

\section{References}

Amin, A. and Thrift, N. (2002) Cities: reimagining the urban, Polity Press, Cambridge, UK.

Boal, A. (1992) Games for actors and non-actors, Routledge, London and New York.

Brandt, E. (2006) 'Designing exploratory design games: a framework for participation in participatory design?', Proceedings of the Ninth Conference on Participatory Design: Expanding Boundaries in Design, Volume 1, Trento, Italy, ACM Press, pp. 57-66.

Butler, J. P. (1989) Gender trouble: feminism and the subversion of identity, Routledge, New York.

Certeau, M. D. (2002 [1984]) The practice of everyday life, trans. S. Rendall, University of California Press, Berkeley, Calif.

CuteCircuit (2006) Hugshirt (f+r hugs), [online], http://www.cutecircuit.com/now/projects/wearables/fr-hugs/, accessed October 222006.

Damasio, A. R. (1994) Descartes'error: emotion, reason, and the human brain, G.P. Putnam, New York.

Dindler, C., Eriksson, E., Iversen, O. S., LykkeOlesen, A. and Ludvigsen, M. (2005) 'Mission from Mars: a method for exploring user requirements for children in a narrative space', Proceed ing of the 2005 Conference on Interaction Design and Children, Boulder, Colorado.

Dunne, A. and Raby, F. (2001) Design noir: the secret life of electronic objects, Birkhäuser, London and Basel.

Gaver, B., Dunne, T. and Pacenti, E. (1999) 'Design: cultural probes', Interactions, vol. 6, no. 1, pp. 21-29.

Greenbaum, J. and Kyng, M. (1991) Design at work. Cooperative design of computer systems, Lawrence Erlbaum Associates, Hillsdale, N.J.

Halskov, K. and Dalsgård, P. (2006) 'Inspiration card workshops', Proceedings of the 6th ACM Conference on Designing Interactive Systems, University Park, PA, USA, ACM Press, pp. 2-11.
Haque, U. (2002) Hardspace, softspace and the possibilities of open source architecture, [online], http:/haque.co.uk/papers/hardsp-softsp-open-soarch.PDF, accessed October 222006.

HP (2006) wireless hub in a watch, [online], http://news.zdnet.co.uk/communications/wireless/0,39020348,39283926,00.htm, accessed October 92006.

Hutchinson, H., Mackay, W., Westerlund, B., Bederson, B. B., Druin, A., Plaisant, C. et al. (2003) 'Technology probes: inspiring design for and with families', Proceedings of the SIGCHI Conference on Human Factors in Computing Systems, Ft. Lauderdale, Florida, USA.

Kozel, S. (2006) 'Revealing practices: Heidegger's techne interpreted through performance in responsive systems', Performance Research, vol. 10, no. 4.

Kozel, S. (in print 2007) Closer: performance, technologies, phenomenology, The MIT Press, Cambridge, Massachusetts.

Merleau-Ponty, M. (1987 [1964]) The visible and the invisible, trans. A. Lingis, Northwestern University Press, Evanston.

Moen, J. (2005) 'Towards people based movement interaction and kinaesthetic interaction experiences', Proceedings of the 4th Decennial Conference on Critical Computing: between Sense and Sensibility, Aarhus, Denmark.

Muller, M. J. (2003) 'Participatory design: the third space in HCI' in The human-computer interaction handbook: fundamentals, evolving technologies and emerging applications, eds J.A. Jacko and A. Sears, Lawrence Erlbaum Associates, Inc, pp. 1051-1068.

Read, A. (1995) Theatre and everyday life: an ethics of performance, Routledge, London and New York.

Rheingold, H. (2002) Smart mobs: the next social revolution, Perseus, Cambridge, Massachusetts.

Schechner, R. (2003 [1988]) Performance theory, 2nd ed, Routledge, London.

Schiphorst, T. (2006) 'Affectionate computing: can we fall in love with a machine?', IEEE MultiMedia, vol. 13, no. 1, pp. 20-23.

Schiphorst, T. and Andersen, K. (2004) 'Between bodies: using experience modeling to create 
gestural protocols for physiological data transfer', CHI Fringe, ACM CHI 2004, Vienna, Austria.

Sterling, B. (2005) Shaping things, MIT Press, Cambridge, Massachusetts and London, England.

The Toy (2006) [online], http://www.thetoy.co.uk, accessed October 222006.

Varela, F. J., Thompson, E. and Rosch, E. (1993 [1991]) The embodied mind: cognitive science and human experience, MIT Press, Cambridge, Mass.

Varela, Francisco J. (1999 [1992]) Ethical knowhow: action, wisdom and cognition, Stanford University Press, Stanford, CA.

Lone Koefoed Hansen is a researcher working within the area of interface culture, art and experience design and is presently focused on pervasive technologies seen from a cultural and aesthetic perspective. She is currently a $\mathrm{PhD}$ candidate in Multimedia at the Institute of Aesthetic Studies at University of Aarhus (Denmark). She is a member of the research initiative The Aesthetics of Interface Culture and a board member of the Digital Aesthetics Research Centre.

Susan Kozel is a dancer, choreographer and writer working at the interface between digital technologies and moving bodies. She is an Associate Professor at the School of Interactive Arts and Technology at Simon Fraser University (Canada). Her artistic collaborations result in performances, installations and phenomenological writings. She is the director of mesh performance practices (http://www. meshperformance.org). Her book Closer: performance, technologies, phenomenology published by The MIT Press will be released in Autumn 2007. 